Discrete Comput Geom 37:565-576 (2007)

DOI: $10.1007 / \mathrm{s} 00454-006-1286-3$

\title{
Open Caps and Cups in Planar Point Sets*
}

\author{
Pavel Valtr \\ Department of Applied Mathematics and \\ Institute for Theoretical Computer Science (ITI), Charles University, \\ Malostranské nám. 25, 11800 Praha 1, Czech Republic \\ http://kam.mff.cuni.cz/ valtr
}

\begin{abstract}
Points $p_{1}, p_{2}, \ldots, p_{k}$ in the plane, ordered in the $x$-direction, form a $k$-cap ( $k$-cup, respectively) if they are in convex position and $p_{2}, \ldots, p_{k-1}$ lie all above (below, respectively) the segment $p_{1} p_{k}$. We prove the following generalization of the Erdôs-Szekeres theorem. For any $k$, any sufficiently large set $P$ of points in general position contains $k$ points, $p_{1}, p_{2}, \ldots, p_{k}$, that form either a $k$-cap or a $k$-cup, and there is no point of $P$ vertically above the polygonal line $p_{1} p_{2} \cdots p_{k}$. We give double-exponential lower and upper bounds on the minimal size of $P$. We also give several related results.
\end{abstract}

\section{Introduction}

A configuration is a finite set of points in the plane such that no three points lie on a line and no two points have the same $x$-coordinate. Let $X$ be a configuration. We say that a subset of $X$ is in convex position, if it is the vertex set of a convex polygon. Let $p_{1}, \ldots, p_{k}$ be $k$ points of $X$ ordered increasingly with respect to their $x$-coordinates (i.e., $\left.x\left(p_{1}\right)<x\left(p_{2}\right)<\cdots<x\left(p_{k}\right)\right)$. For $i=1, \ldots, k-1$, let $s_{i}$ be the slope of the line $p_{i} p_{i+1}$, i.e., $s_{i}=\left(y\left(p_{i+1}\right)-y\left(p_{i}\right)\right) /\left(x\left(p_{i+1}\right)-x\left(p_{i}\right)\right)$. The set $P=\left\{p_{1}, \ldots, p_{k}\right\}$ is a $k$-cap or a $k$-cup if the sequence $s_{1}, s_{2}, \ldots, s_{k-1}$ is decreasing or increasing, respectively (see Fig. 1). The set $P=\left\{p_{1}, \ldots, p_{k}\right\}$ is open (in $\left.X\right)$, if no point $p \in X$ with $x\left(p_{1}\right)<x(p)<x\left(p_{k}\right)$ lies above the polygonal line $p_{1} p_{2} \cdots p_{k}$.

In 1935 Erdős and Szekeres proved the following classical result:

Theorem 1 [3]. For any $n \geq 3$, there is a (least) integer $F(n)$ such that any set of at least $F(n)$ points in general position in the plane contains $n$ points in convex position.

\footnotetext{
* This research was supported by projects LN00A056 and 1M0021620808 of The Ministry of Education of the Czech Republic.
} 


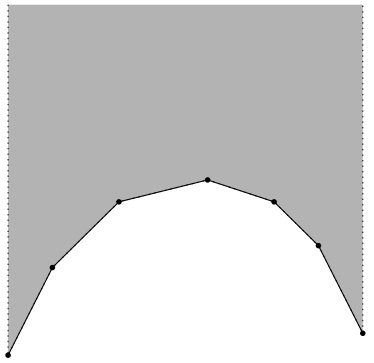

7-cap

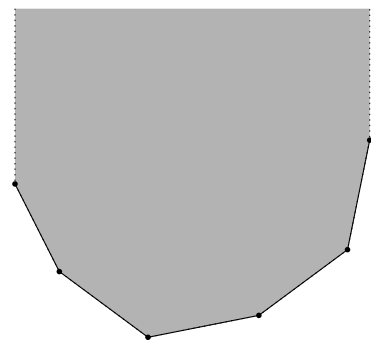

6-cup

Fig. 1. A 7-cap and a 6-cup; each of them is open if the unbounded shaded region contains no points of $X$.

Erdős and Szekeres [3] proved Theorem 1 by proving the following result:

Theorem 2 [3]. For any $k, l \geq 2$, there is a (least) integer $F^{\prime}(k, l)$ such that any configuration at least $F^{\prime}(k, l)$ points contains a $k$-cap or an l-cup.

Erdős and Szekeres [3] proved that

$$
F^{\prime}(k, l)=\left(\begin{array}{c}
k+l-4 \\
k-2
\end{array}\right)+1 \quad \text { for any } \quad k, l \geq 2 .
$$

It follows that

$$
F(n) \leq F^{\prime}(n, n)=\left(\begin{array}{c}
2 n-4 \\
n-2
\end{array}\right)+1 \quad \text { for any } n \geq 3 .
$$

It is known that $F(3)=3, F(4)=5$, and $F(5)=9$. For $n>5$, the best known bounds are

$$
2^{n-2}+1 \leq F(n) \leq\left(\begin{array}{c}
2 n-5 \\
n-2
\end{array}\right)+1
$$

(see [4] and [12]).

Here we show the following generalization of Theorem 2:

Theorem 3. For any $k, l \geq 2$, there is a (least) integer $f(k, l)$ such that any configuration of size at least $f(k, l)$ contains an open $k$-cap or an open $l$-cup.

Theorem 3 is a "generic" result with some interesting corollaries mentioned below. The function $f(k, l)$ is double-exponential, as shown in Theorem 4 below.

If $X$ is a configuration, then a subset $P \subseteq X$ in convex position is called an empty polygon (in $X$ ) if the interior of conv $P$ contains no point of $X$. Answering a question of Erdős [2], Horton [6] constructed arbitrarily large configurations with no empty 7-gon. Gerken [5] has shown very recently that any sufficiently large configuration contains 
an empty hexagon [5]. A modification of Gerken's proof can be found in [16]. Another proof is given in [11].

Our results show some structural properties of configurations containing no empty polygons with many vertices. We say that a configuration $X$ is $k$-convex if the interior of every triangle determined by $X$ contains at most $k$ points of $X$. Theorem 3 implies the following result which shows that large $k$-convex configurations contain large empty polygons:

Corollary 1 [15], [9]. For any $k \geq 1$ and $l \geq 3$, there is a (least) integer $N(k, l)$ such that any $k$-convex configuration of size at least $N(k, l)$ contains an empty $l$-gon.

Corollary 1 in the special case $k=1$ was first proved by Károlyi et al. [7]. In case $k=$ $1, N(1, l)$ is exponential in $l$ [9] and its exact value is determined in [8]. For general $k$ and $l$, our proof of Corollary 1 gives a double-exponential upper bound $N(k, l) \leq 2^{\left(\begin{array}{l}k+l \\ k+2\end{array}\right)-1}+1$, which is slightly better than the previous bound $N(k, l) \leq(k+2)^{(k+2)^{l}-1} \leq 2^{(k+2)^{l+1}}$ of Kun and Lippner [9]. No lower bound on $N(k, l)$ better than exponential in $k+l$ is known.

We asked in [15] if any large configuration with no $k$-cap contains a large empty polygon. This is answered affirmatively by the following direct consequence of Theorem 3 :

Corollary 2. For any $k, l \geq 2$, there is a least integer $m(k, l)$ such that any configuration of size at least $m(k, l)$ with no open $k$-cap contains an empty $l$-gon.

A $k$-moon is a $k$-point configuration $M$ with a specific point $a(M) \in M$ (called the apex of $M$ ) such that any 4-point subset of $M$ is in convex position if and only if it does not contain $a(M)$. The combinatorial structure of a $k$-moon is unique for each $k \geq 3$ (see Fig. 2). A $k$-moon $M$ is empty (in $X$ ), if the interior of the region conv $M \backslash \operatorname{conv}(M \backslash\{a(M)\})$ contains no point of $X$ (see Fig. 2). Theorem 3 is equivalent to the following corollary:

Corollary 3. For any $k, l \geq 3$, there is a least integer $z(k, l)$ such that any point $p$ in any configuration $X$ of size at least $z(k, l)$ is the apex of an empty $k$-moon in $X$ or it is one of the vertices of an empty l-gon in $X$.

We obtain double-exponential lower and upper bounds on $f(k, l)$ and on two related functions:

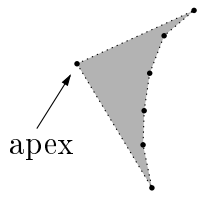

Fig. 2. A 7-moon; it is empty in $X$ if the shaded region contains no points of $X$. 
Theorem 4. Let $k, l \geq 2$ and let $f(k, l)$ be the number given by Theorem 3. Then:

(i) If $f^{\prime}(k, l)$ denotes the minimum integer such that any configuration of size at least $f^{\prime}(k, l)$ contains a $k$-cap or an open $l$-cup, then

$$
2^{\left(\begin{array}{c}
{[k / 2]+[l / 2\rfloor-2} \\
\lfloor/ 2]-1
\end{array}\right)} \leq f^{\prime}(k, l) \leq f(k, l) \leq 2^{\left(\begin{array}{c}
k+l-2 \\
k-1
\end{array}\right)-1} .
$$

(ii) If $f^{\prime \prime}(k, l)$ denotes the minimum integer such that any configuration of size at least $f^{\prime \prime}(k, l)$ contains an open $k$-cap or an $l$-cup, then

$$
2^{\left(\frac{k k / 2]+[l-1) / 2\rfloor-2}{\lfloor k / 2]-1}\right)} \leq f^{\prime \prime}(k, l) \leq f(k, l) .
$$

If neither the $k$-cap nor the $l$-cup are required to be open, then the extremal function is exponential (see Theorem 2 and the remark below it).

Our proof of Theorem 3 uses some of the ideas of Kun and Lippner [9]. It also has some similarities with one of the original proofs of the Erdôs-Szekeres theorem (Theorem 1). A different (and shorter) proof of Theorem 3 has been very recently proved by Černý [1].

Open Problems. Corollaries 1-3 can be seen as a little step towards an answer to the following somewhat loosely stated problem:

Problem 1. Which types of subconfigurations are contained in any sufficiently large configuration with no empty $l$-gon $(l \geq 7$ fixed)?

Since the so-called Horton sets (e.g., see [13], [14], and [10]) contain no empty $7-$ gons, Problem 1 may be restricted to types of configurations contained in all sufficiently large Horton sets.

\section{Proof of Theorem 3}

We write $C=c_{1} c_{2} \cdots c_{k}$ if $C=\left\{c_{1}, c_{2}, \ldots, c_{k}\right\}$ is a $k$-cap (or a $k$-cup) with $x\left(c_{1}\right)<$ $x\left(c_{2}\right)<\cdots<x\left(c_{k}\right)$. If $x(p)<x(q)$ then we say that $p$ lies to the left of $q$ and $q$ lies to the right of $p$. If $x(p)<x(q)<x(r)$ then we say that $q$ lies between $p$ and $r$. The left strip of an $l$-cup $D=d_{1} d_{2} \cdots d_{l}$ is the vertical strip $L(D)=\left\{p \in \mathbb{R}^{2}: x\left(d_{1}\right)<\right.$ $\left.x(p)<x\left(d_{2}\right)\right\}$ (see Fig. 3). A right strip of a $k$-cap $C=c_{1} c_{2} \cdots c_{k}$ is any vertical strip $R(C, w)=\left\{p \in \mathbb{R}^{2}: x\left(c_{k}\right)<x(p)<x\left(c_{k}\right)+w\right\}$, where $w \in \mathbb{R}^{+}$. We further define $R^{-}(C, w)$ as the set of points of $R(C, w)$ lying below the line $c_{k-1} c_{k}$ (see Fig. 3).

For $\varepsilon>0$ and for a configuration $X$, we say that an $l$-cup $D, D \subseteq X$, is $\varepsilon$-good (in $X$ ) if it is open and $|X \cap L(D)| \geq \varepsilon|X|-1$ (see Fig. 4). We say that a $k$-cap $C$, $C \subseteq X$, is $\varepsilon-\operatorname{good}($ in $X$ ) if it is open and there is a $w>0$ such that $|X \cap R(C, w)|=$ $\left|X \cap R^{-}(C, w)\right| \geq \varepsilon|X|-1$ (see Fig. 4).

For $k, l \geq 2$, we recursively define a parameter $\varepsilon(k, l) \in\left(0, \frac{1}{2}\right]$ as follows:

(i) $\varepsilon(i, 2)=\varepsilon(2, i)=1 / i$ for $i \geq 2$,

(ii) $\varepsilon(k, l)=\varepsilon(k-1, l) \cdot \varepsilon(k, l-1) / 2$ for $k, l \geq 3$. 

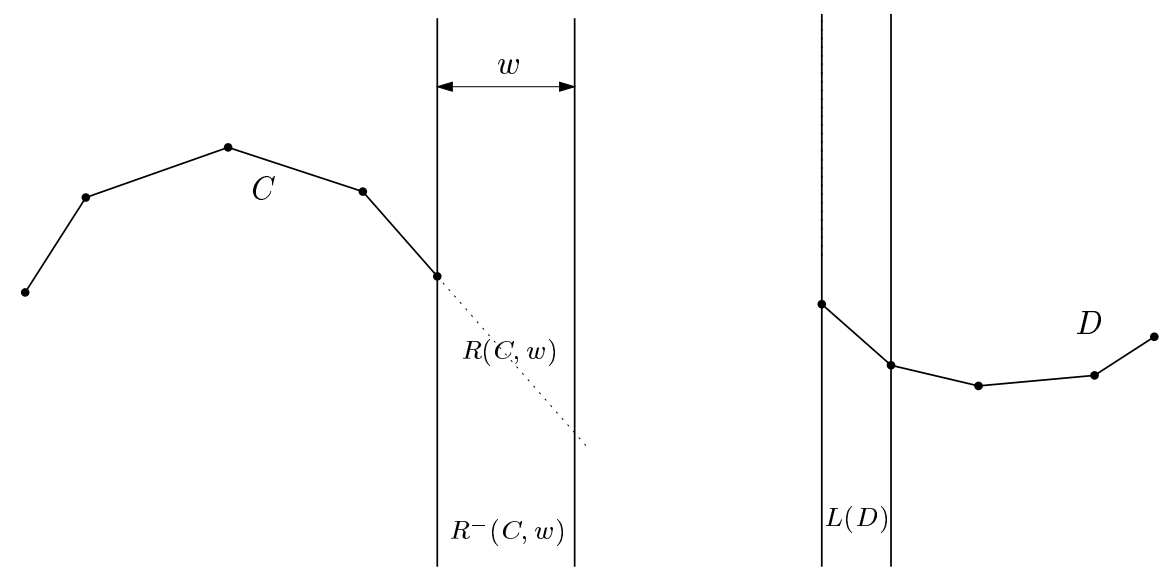

Fig. 3. The regions $R(C, w), R^{-}(C, w)$, and $L(D)$.

We prove Theorem 3 by proving the following statement:

(*) For any $k, l \geq 2$, any configuration $X$ of size at least $1 / \varepsilon(k, l)$ contains an $\varepsilon(k, l)$-good $k$-cap or an $\varepsilon(k, l)$-good $l$-cup.

First we verify $(*)$ for $k=2, l \geq 2$ and for $l=2, k \geq 2$, and then continue by induction on $k+l$. The case $m=l$ in the following lemma gives $(*)$ for $k=2, l \geq 2$.

Lemma 1. Let $X$ be a configuration of size at least $l \geq 2$ with no $1 / l$-good 2-cap. Then for each $m=2,3, \ldots, l$, the set $X$ contains a $1 / l$-good $m$-cup $D_{m}=d_{1} d_{2} \cdots d_{m}$ such that fewer than $m|X| / l$ points of $X$ lie to the left of $d_{m}$.

Proof. First let $m=2$. Let $d_{1}$ be the leftmost point of $X$, and let $X_{0}$ be the set of $\lceil 2|X| / l\rceil$ leftmost points of $X$. Further, let $d_{2}$ be the point viewed from $d_{1}$ as the highest

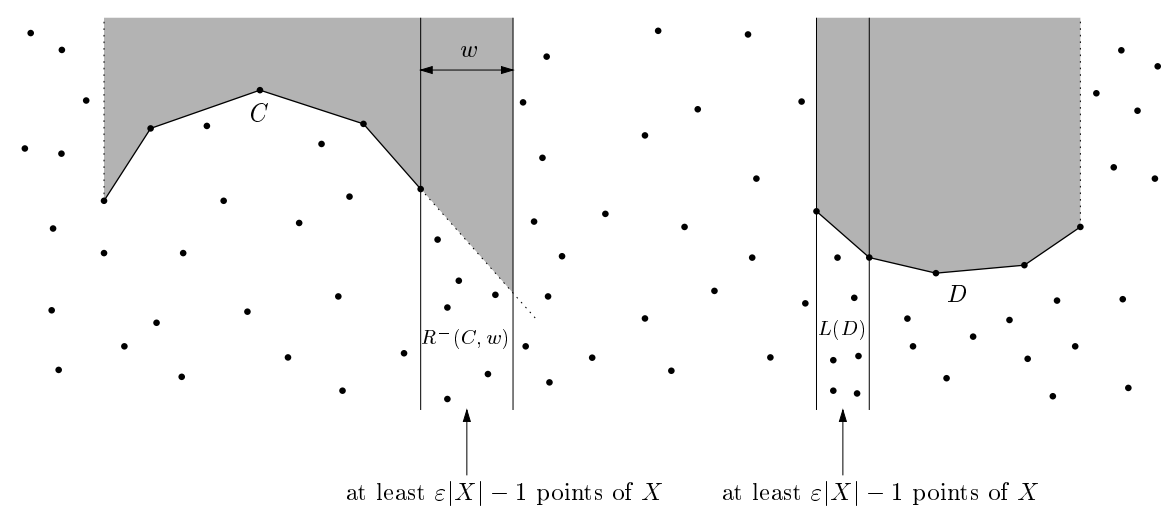

Fig. 4. An $\varepsilon$-good 5-cap and an $\varepsilon$-good 5-cup (the shaded regions contain no point of $X$ ). 


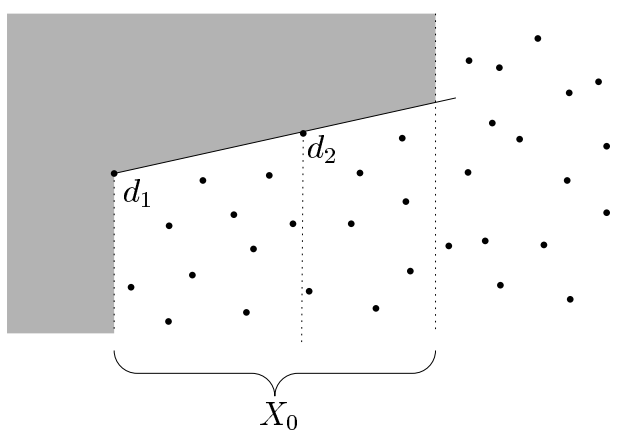

Fig. 5. The points $d_{1}$ and $d_{2}$ (the shaded region contains no point of $X$ ).

point of $X_{0} \backslash\left\{d_{1}\right\}$. Thus, $d_{2} \in X_{0} \backslash\left\{d_{1}\right\}$ and no point of $X_{0}$ lies above the line $d_{1} d_{2}$ (see Fig. 5). Since the open 2-cap $d_{1} d_{2}$ is not $1 / l$-good in $X$, fewer than $|X| / l-1$ points of $X_{0}$ lie to the right of $d_{2}$. It follows that at least $|X| / l-1$ points of $X_{0}$ lie between $d_{1}$ and $d_{2}$ (recall that the size of $X_{0}$ is $\lceil 2|X| / l\rceil$ ). Thus, the 2-cup $D_{2}=d_{1} d_{2}$ is $1 / l$-good in $X$ and fewer than $2|X| / l$ points of $X$ lie to the left of $d_{2}$. This settles the case $m=2$.

We further proceed by induction on $m$. Let $2 \leq m<l$ and let $D_{m}=d_{1} d_{2} \cdots d_{m}$ be a $1 / l$-good $m$-cup $D_{m}=d_{1} d_{2} \cdots d_{m}$ such that fewer than $m|X| / l$ points of $X$ lie to the left of $d_{m}$. Let $d_{m+1}$ be the leftmost point of $X$ lying to the right of $d_{m}$ and above the line $d_{m-1} d_{m}$ (see Fig. 6). Such a point $d_{m+1}$ exists, since otherwise the 2-cap $d_{m-1} d_{m}$ would be $1 / l$-good (at least $|X|-m|X| / l-1 \geq|X| / l-1$ points of $X$ lie to the right of $d_{m}$ ). For the same reason fewer than $|X| / l-1$ points of $X$ lie between $d_{m}$ and $d_{m+1}$. It follows that fewer than $m|X| / l+1+(|X| / l-1)=(m+1)|X| / l$ points of $X$ lie to the left of $d_{m+1}$. Since the $(m+1)$-cup $D_{m+1}=d_{1} d_{2} \cdots d_{m+1}$ is $1 / l$-good, this finishes the inductive step from $m$ to $m+1$.

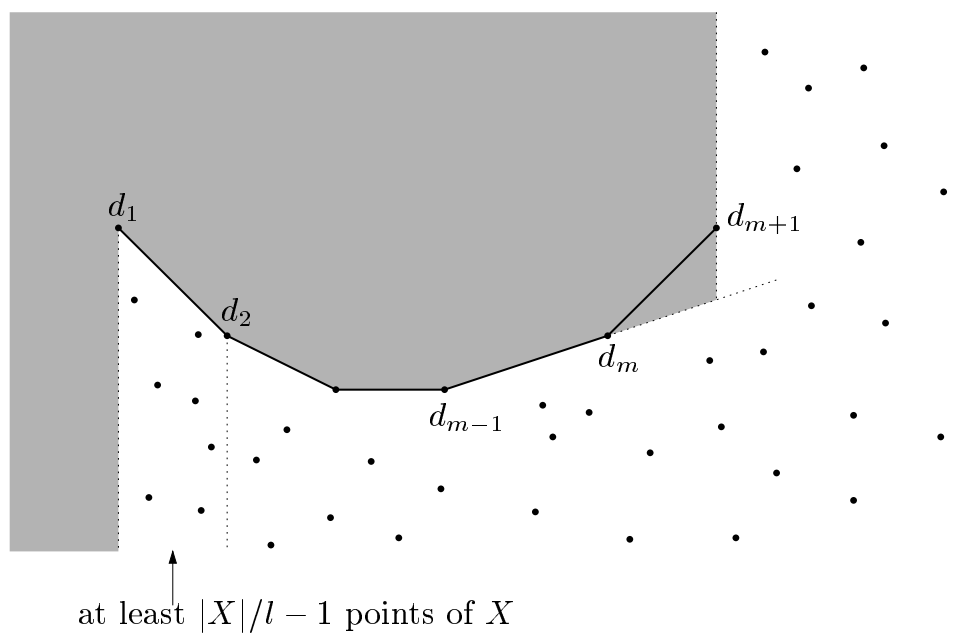

Fig. 6. The $(m+1)$-cup $D_{m+1}=d_{1} d_{2} \cdots d_{m+1}$. 


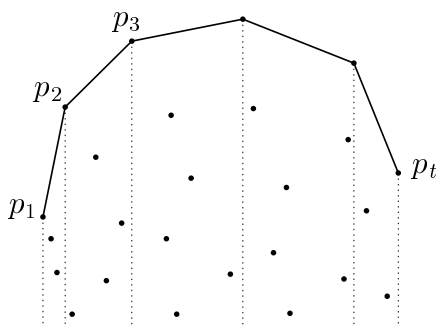

Fig. 7. The upper envelope of $X$.

We now prove $(*)$ for $l=2, k \geq 2$. Let $|X| \geq k$, and let $p_{1}, p_{2}, \ldots, p_{t}, x\left(p_{1}\right)<$ $x\left(p_{2}\right)<\cdots<x\left(p_{t}\right)$, be the vertices of the upper envelope of conv $X$ (see Fig. 7). If $X$ contains at least $|X| / k-1$ points $p$ with $x\left(p_{i}\right)<x(p)<x\left(p_{i+1}\right)$ for some $i$, then the 2 -cup $p_{i} p_{i+1}$ is $1 / k$-good. Otherwise $t>k$ and there are more than $|X| / k-1$ points of $X$ to the right of $p_{k}$. Thus, $p_{1} p_{2} \cdots p_{k}$ is a $1 / k$-good $k$-cap in this case.

It remains to derive $(*)$ for $k, l \geq 3$ from $(*)$ for all $k^{\prime}, l^{\prime} \geq 2, k^{\prime}+l^{\prime}<k+l$. Suppose that $k, l \geq 3$ and that $|X| \geq 1 / \varepsilon(k, l)$. We want to prove that $X$ contains an $\varepsilon(k, l)$-good $k$-cap or an $\varepsilon(k, l)$-good $l$-cup. By the inductive hypothesis, $X$ contains an $\varepsilon(k-1, l)$-good $(k-1)$-cap or an $\varepsilon(k-1, l)$-good $l$-cup. In the second case we are done, since $\varepsilon(k-1, l)>\varepsilon(k, l)$. Thus, assume that $X$ contains an $\varepsilon(k-1, l)$-good $(k-1)$-cap $C=c_{1} c_{2} \cdots c_{k-1}$. Let $X_{0}$ be the set of $\lceil\varepsilon(k-1, l)|X|-1\rceil$ leftmost points of $X$ lying to the right of $c_{k-1}$. Since $C$ is $\varepsilon(k-1, l)$-good, all points of $X_{0}$ lie under the line $c_{k-2} c_{k-1}$.

Since $\lceil\alpha-1\rceil \geq(\lceil\alpha / 4\rceil-1)+(\lceil\alpha / 4\rceil-2)+\lceil\alpha / 2\rceil$ holds $^{1}$ for any $\alpha \in \mathbb{R}$, we can partition the set $X_{0}$ into three subsets $R, S, T$ of sizes

$$
\begin{aligned}
|R| & =\left\lceil\frac{\varepsilon(k-1, l)}{4}|X|\right\rceil-1>2 \cdot \varepsilon(k, l-1) \cdot \frac{\varepsilon(k-1, l)}{4}|X|-1=\varepsilon(k, l)|X|-1, \\
|S| & =\left\lceil\frac{\varepsilon(k-1, l)}{4}|X|\right\rceil-2>\varepsilon(k, l)|X|-2, \\
|T| & \geq\left\lceil\frac{\varepsilon(k-1, l)}{2}|X|\right\rceil
\end{aligned}
$$

(in the estimate of $|R|$ we used that $2 \cdot \varepsilon(k, l-1)<1$ for any $k, l \geq 3$ ). The partition is done "from left to right" so that $x(r)<x(s)<x(t)$ holds for any $r \in R, s \in S, t \in T$.

Since

$$
|T| \geq \frac{\varepsilon(k-1, l)}{2}|X| \geq \frac{\varepsilon(k-1, l)}{2} \cdot \frac{1}{\varepsilon(k, l)}=\frac{1}{\varepsilon(k, l-1)},
$$

the set $T$ contains an $\varepsilon(k, l-1)$-good $k$-cap or an $\varepsilon(k, l-1)$-good $(l-1)$-cup. In the first case we are done since $|T| \geq(\varepsilon(k-1, l) / 2)|X|$ and therefore any $k$-cap which is $\varepsilon(k, l-1)$-good in $T$ is $\varepsilon(k, l-1) \cdot(\varepsilon(k-1, l) / 2)=\varepsilon(k, l)$-good in $X$. So we

\footnotetext{
${ }^{1}$ The inequality holds with equality for $\alpha=4 m+q, m \in \mathbf{Z}, q \in(0,1]$. Otherwise the left-hand side is larger than the right-hand side.
} 


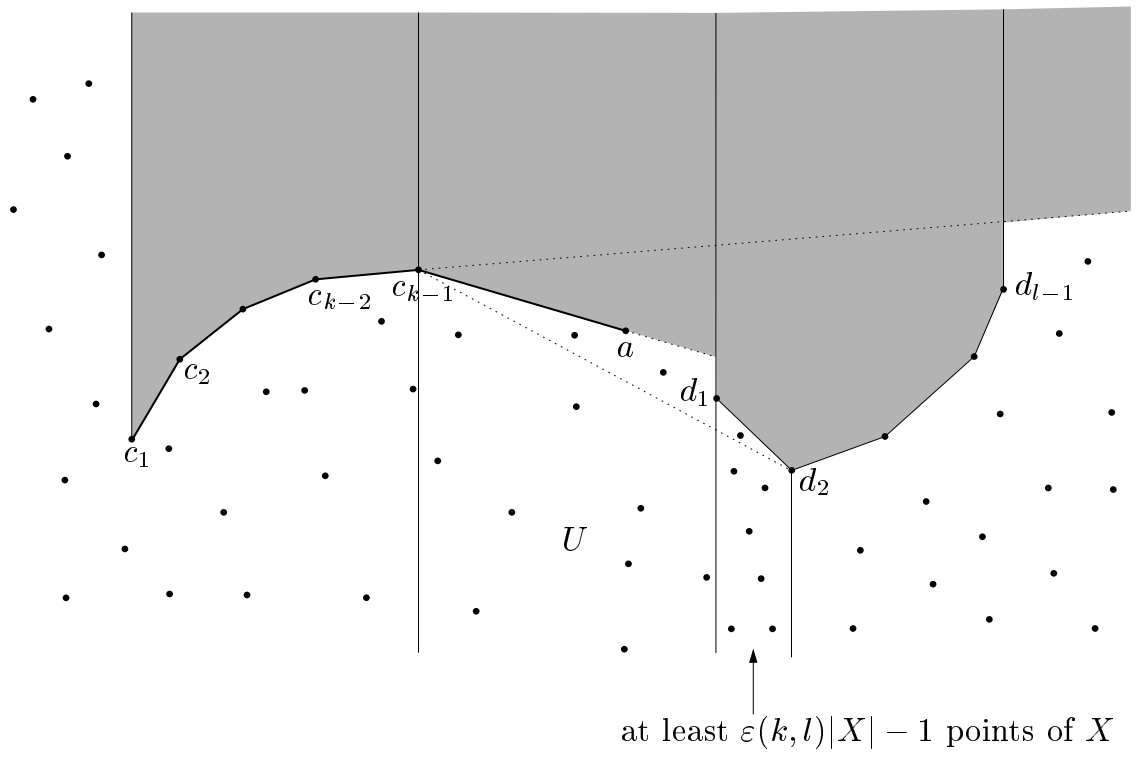

Fig. 8. Case 1 (the shaded regions contain no points of $X$ ).

may suppose that $T$ contains an $\varepsilon(k, l-1)$-good $(l-1)$-cup $D=d_{1} d_{2} \cdots d_{l-1}$. Since $|T| \geq(\varepsilon(k-1, l) / 2)|X|, D$ is $\varepsilon(k, l-1) \cdot(\varepsilon(k-1, l) / 2)=\varepsilon(k, l)$-good in $X$.

Let $U$ be the set of points $p$ of $X_{0}$ with $x(p) \leq x\left(d_{1}\right)$. Let $a$ be the point viewed from $c_{k-1}$ as the highest point of $U$ (see Fig. 8). In other words, $a \in U$ and no point of $U$ lies above the line $c_{k-1} a$. We now distinguish three cases.

Case 1: a lies above the line $c_{k-1} d_{2}$ (see Fig. 8). In this case, $c_{1} c_{2} \cdots c_{k-1} a$ is an $\varepsilon(k, l)$ $\operatorname{good} k$-cap in $X$ (recall that $D$ is $\varepsilon(k, l)$-good in $X$ and thus $|L(D) \cap X| \geq \varepsilon(k, l)|X|-1)$.

Case 2: a lies below the line $c_{k-1} d_{2}$ and in $R$ (see Fig. 9). In this case, $c_{1} c_{2} \cdots c_{k-1} a$ is an $\varepsilon(k, l)$-good $k$-cap in $X\left(S \cup\left\{d_{1}\right\}\right.$ lies entirely below the line $\left.c_{k-1} a\right)$.

Case 3: a lies below the line $c_{k-1} d_{2}$ and in $U \backslash R$ (see Fig. 10). In this case, $c_{k-1} a d_{2} d_{3} \cdots d_{l-1}$ is an $\varepsilon(k, l)$-good $l$-cup in $X$ ( $R$ lies entirely below the segment $\left.c_{k-1} a\right)$.

This concludes the proof of Theorem 3.

\section{Proof of Corollaries 1-3}

Proof of Corollary 1. Let $X$ be a configuration of size at least $f(k+3, l-1)+1$. Let $p$ be a vertex of conv $X$, and let $l$ be a line touching conv $X$ at the point $p$. We transform $X$ to a set $X^{\prime}$ by a projective transformation $P$ which sends the point $p$ to the "point" $(0, \infty)$ and the line $l$ to the line at infinity. The configuration $X^{\prime}$ has at least $f(k+3, l-1)$ points in the real plane, thus it contains an open $(k+3)$-cap $C$ or an open $(l-1)$-cup $D$. 


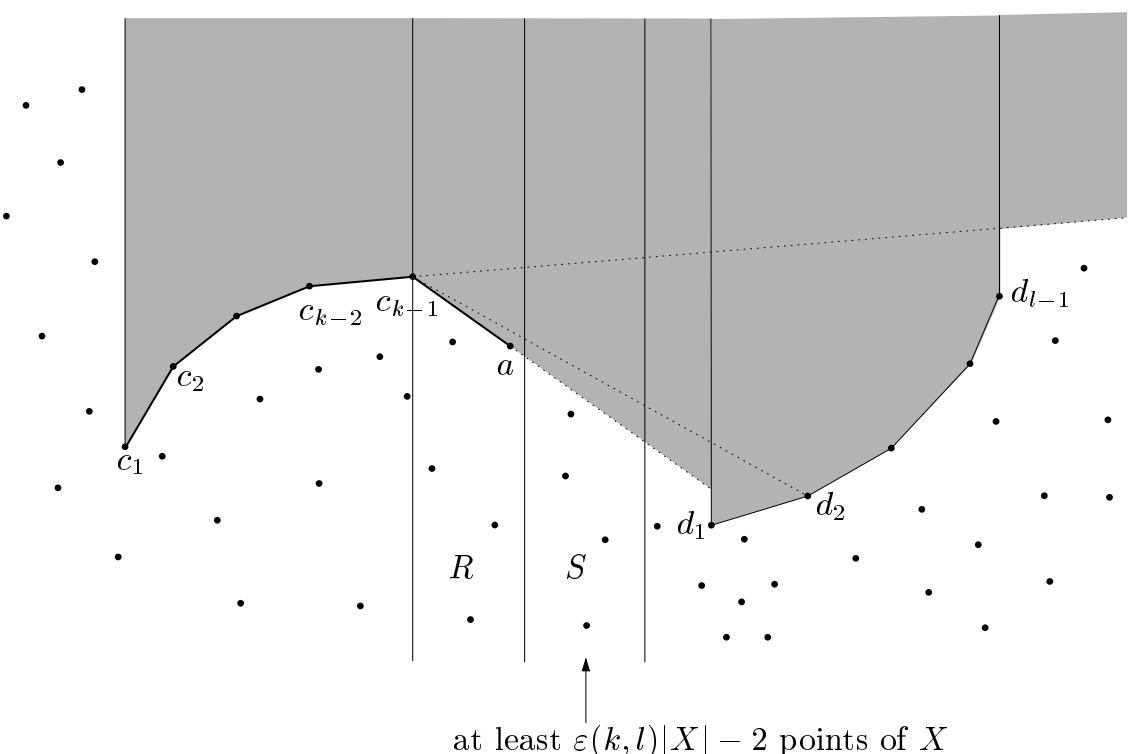

Fig. 9. Case 2.

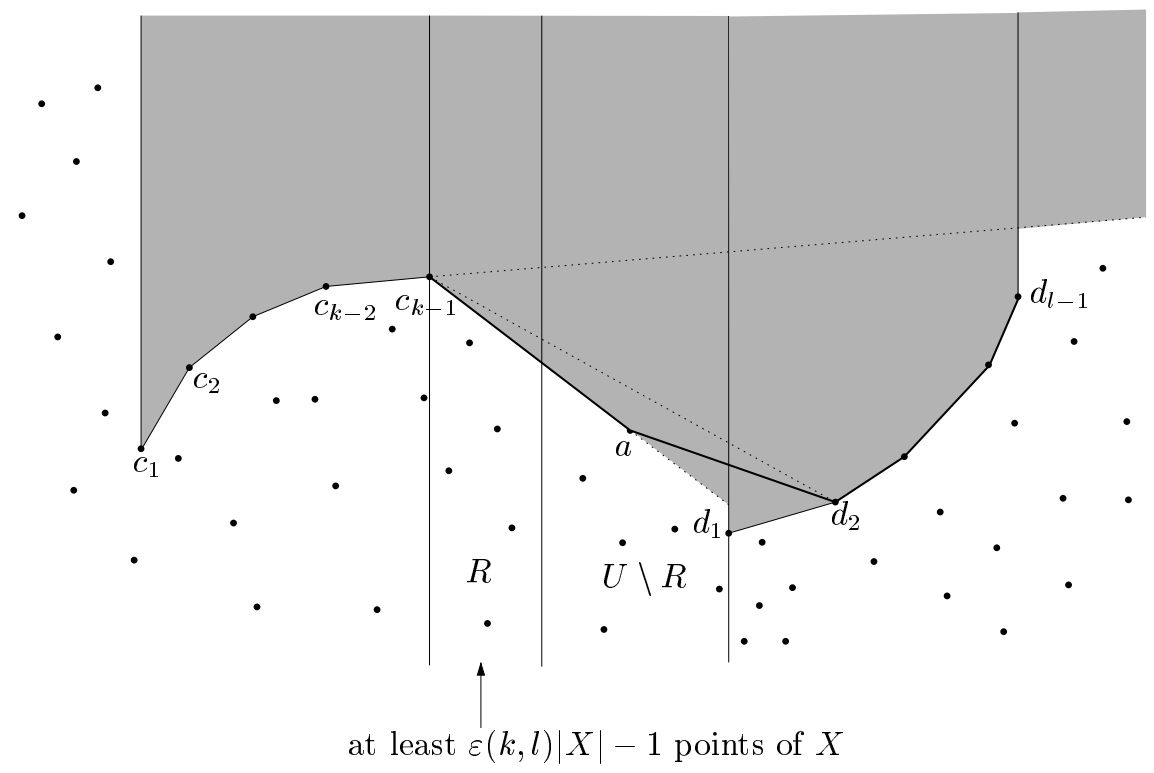

Fig. 10. Case 3. 
The first case cannot happen, since $P^{-1}(C) \cup\{p\}$ would be an (empty) $(k+4)$-moon and we would get a contradiction with the $k$-convexity of $X$. In the second case the set $P^{-1}(D) \cup\{p\}$ forms an empty $l$-gon. Thus, $N(k, l) \leq f(k+3, l-1)+1$.

Proof of Corollary 2. Since any open $l$-cup is an empty $l$-gon, Theorem 3 immediately gives $m(k, l) \leq f(k, l)$.

Proof of Corollary 3. If a configuration $X$ has at least $2 \cdot f(k-1, l-1)$ points and $p \in X$ is any point in $X$, then $p$ is a vertex of the convex hull of at least $f(k-1, l-1)+1$ points of $X$. We further continue analogously as in the proof of Corollary 1, obtaining $z(k, l) \leq 2 \cdot f(k-1, l-1)$.

\section{Proof of Theorem 4}

Proof of Theorem 4. The inequalities $f^{\prime}(k, l) \leq f(k, l), f^{\prime \prime}(k, l) \leq f(k, l)$ are trivial, and the proof of Theorem 3 gives the upper bound in (i):

$$
f(k, l) \leq \frac{1}{\varepsilon(k, l)} \leq 2^{\left(\begin{array}{c}
k+l-2 \\
k-1
\end{array}\right)-1}
$$

(the second inequality can be easily proved by induction from the definition of the coefficients $\varepsilon(k, l))$. It remains to prove the lower bounds.

For $k, l \geq 2$ even, we recursively construct a configuration $S_{k, l}$ of size $2^{\left(\begin{array}{c}k / 2+l / 2-2 \\ k / 2-1\end{array}\right)}-1$ with no $k$-cap and no open $l$-cup. This gives the lower bound in (i). Slight changes in the construction will then give the lower bound in (ii).

If $k=2$ or $l=2$ then $S_{k, l}=\{(0,0)\}$. We continue by induction on $k+l$. Let $k, l \geq 4$ be even, and suppose that the configurations $S_{k-2, l}, S_{k, l-2}$ have already been constructed. We take the configuration $S_{k-2, l}$ and partition the plane into $\left|S_{k-2, l}\right|+1$ regions by the vertical lines through each point of $S_{k-2, l}$. In each region we place a small (shrunk) copy of $S_{k, l-2}$. We denote the copies of $S_{k, l-2}$ by $S^{0}, S^{1}, \ldots, S^{\left|S_{k-2, l}\right|}$. In each $S^{i}$ we shrink the $y$-coordinates. Then we rotate and vertically shift each $S^{i}$ so that any subset of $S^{0} \cup \cdots \cup S^{\left|S_{k-2, l}\right|}$ intersecting each $S^{i}$ in at most two points is a $t$-cup for some $t$. Finally, we shift $S^{0} \cup \cdots \cup S^{\left|S_{k-2, l}\right|}$ vertically so far downwards that the following two conditions hold in the set $S_{k, l}:=S_{k-2, l} \cup\left(S^{0} \cup \cdots \cup S^{\left|S_{k-2, l}\right|}\right)$ (see Fig. 11):

(C1) If $C=c_{1} c_{2} \cdots c_{t}$ is a $t$-cap in $S_{k, l}$, then either it is fully contained in one of the configurations $S^{i}$ or $c_{2} c_{3} \ldots c_{t-1}$ is a $(t-2)$-cap in $S_{k-2, l}$.

(C2) If $D=d_{1} d_{2} \cdots d_{t}$ is an open $t$-cup in $S_{k, l}$, then either it is fully contained in $S_{k-2, l}$ or $d_{2} d_{3} \cdots d_{t-1}$ is an open $(t-2)$-cup in one of the configurations $S^{i}$.

It follows from (C1) and (C2) that $S_{k, l}$ contains no $k$-cap and no open $l$-cup. Its size is

$$
\left|S_{k, l}\right|=\left(\left|S_{k-2, l}\right|+1\right)\left(\left|S_{k, l-2}\right|+1\right)-1=2^{\left(\begin{array}{c}
k / 2+l / 2-3 \\
k / 2-2
\end{array}\right)} \cdot 2^{\left(\begin{array}{c}
k / 2+1 / 2-3 \\
k / 2-1
\end{array}\right)}-1=2^{\left(\begin{array}{c}
k / 2+l / 2-2 \\
k / 2-1
\end{array}\right)}-1 .
$$

This gives the lower bound in (i).

To get the lower bound in (ii), we slightly change the recursive step in the construction. For $k, l \geq 2$ even, we now construct a configuration $T_{k, l}$ of size $2^{\left(\begin{array}{c}k / 2+/ 2-2 \\ k / 2-1\end{array}\right)}-1$ with no 


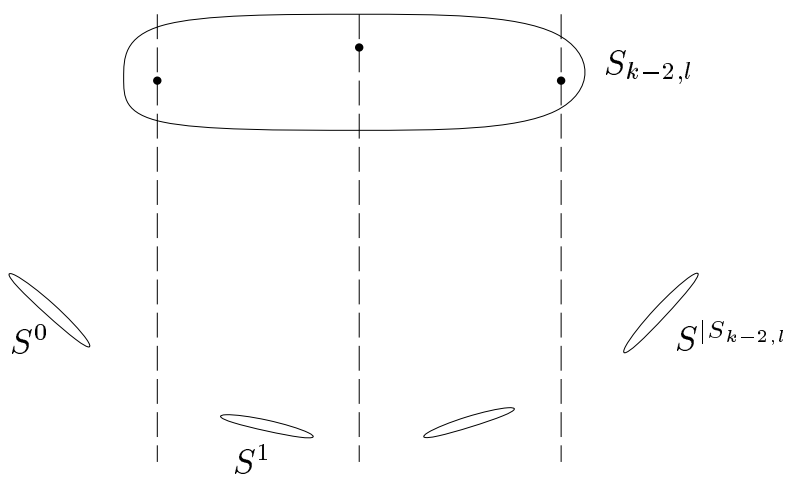

Fig. 11. The configuration $S_{k, l}$ giving the lower bound in Theorem 4(i).

open $k$-cap and no $(l+1)$-cup. Again $T_{k, l}=\{(0,0)\}$ if $\min \{k, l\}=2$. For $k, l \geq 4$ even, the recursive step indicated in Fig. 12 starts similarly as above but the rotations and vertical shifts of the sets $T^{i}$ are done so that the following hold for the set $T_{k, l}:=$ $T_{k-2, l} \cup\left(T^{0} \cup \cdots \cup T^{\left|T_{k-2, l}\right|}\right)$ :

( $\left.\mathrm{Cl}^{\prime}\right)$ If $C=c_{1} c_{2} \cdots c_{t}$ is an open $t$-cap in $T_{k, l}$, then either it is fully contained in one of the configurations $T^{i}$ or $c_{2} c_{3} \cdots c_{t-1}$ is an open $(t-2)$-cap in $T_{k-2, l}$, and

(C2') if $D=d_{1} d_{2} \cdots d_{t}$ is a $t$-cup in $T_{k, l}$ and $t \geq 5$, then either it is fully contained in $T_{k-2, l}$ or $d_{2} d_{3} \cdots d_{t-1}$ is a $(t-2)$-cup in one of the configurations $T^{i}$.

Then the set $T_{k, l}$ of size $2^{\left({ }^{k / 2+l / 2-2}\right)}-1$ indeed contains no open $k$-cap and no $(l+1)$-cup. The lower bound in (ii) follows.

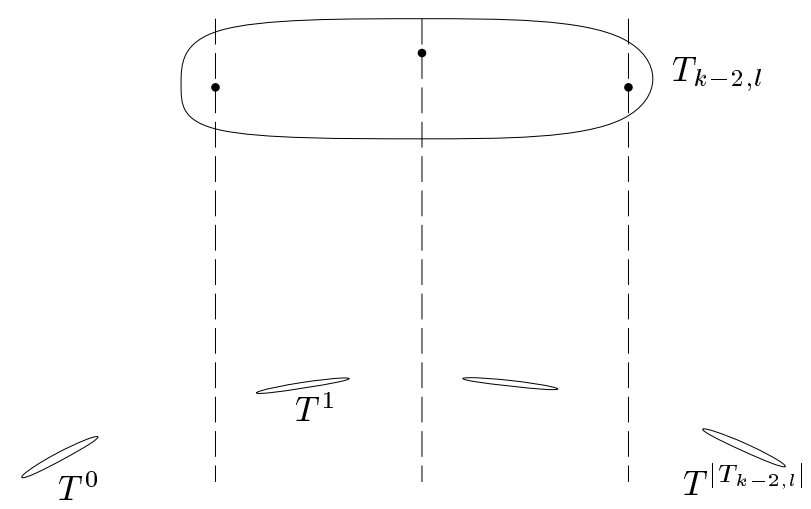

Fig. 12. The configuration $T_{k, l}$ giving the lower bound in Theorem 4(ii). 


\section{Acknowledgment}

I am grateful to Gyula Károlyi, Gábor Lippner, and Helena Nyklová for interesting and stimulating discussions. I also thank two anonymous referees for their helpful comments.

\section{References}

1. J. Černý, A simple proof for open cups and caps, European J. Combin., to appear.

2. P. Erdős, On some problems of elementary and combinatorial geometry, Ann. Mat. Pura. Appl. (4) 103 (1975), 99-108.

3. P. Erdős and Gy. Szekeres, A combinatorial problem in geometry, Compositio Math 2 (1935), 464-470.

4. P. Erdős and Gy. Szekeres, On some extremum problems in elementary geometry, Ann. Univ. Sci. Budapest. Eötvös Sect. Math. 3/4 (1960-61), 53-62.

5. T. Gerken, On empty convex hexagons in planar point sets, to appear.

6. J. D. Horton, Sets with no empty convex 7-gons, Canad. Math. Bull. 26 (1983), 482-484.

7. Gy. Károlyi, J. Pach, and G. Tóth, A modular version of the Erdős-Szekeres theorem, Studia Sci. Math. Hungar. 38 (2001), 245-259.

8. Gy. Károlyi, G. Lippner, and P. Valtr, Empty convex polygons in almost convex sets, Periodica Math. Hungar., to appear.

9. G. Kun and G. Lippner, Large convex empty polygons in k-convex sets, Period. Math. Hungar. 46 (2003), $81-88$.

10. J. Matoušek, Lectures on Discrete Geometry, Springer, Berlin, 2002.

11. C. M. Nicolás, Empty convex hexagons in planar point sets, submitted.

12. G. Tóth and P. Valtr, The Erdôs-Szekeres theorem: upper bounds and related results, in: J. E. Goodman, J. Pach, and E. Welzl (eds.), Combinatorial and Computational Geometry, MSRI Publications 52, 2005, pp. 557-568.

13. P. Valtr, Convex independent sets and 7-holes in restricted planar point sets, Discrete Comput. Geom. 7 (1992), 135-152.

14. P. Valtr, On the minimum number of empty polygons in planar point sets, Studia Sci. Math. Hungar. 30 (1995), 155-163.

15. P. Valtr, A sufficient condition for the existence of large empty convex polygons, Discrete Comput. Geom. 28 (2002), 671-682.

16. P. Valtr, On the empty hexagon theorem, to appear.

Received May 4, 2004, and in revised form June 8, 2006. Online publication April 17, 2007. 